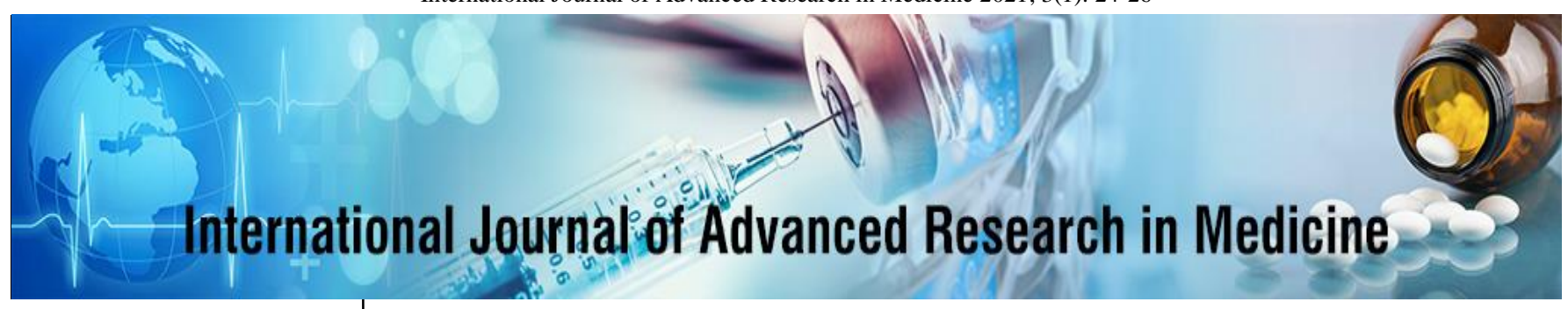

E-ISSN: 2706-9575 P-ISSN: 2706-9567 IJARM 2021; 3(1): 24-26 Received: 03-01-2021 Accepted: 28-01-2021

Dr. Varalakshmidevi B Assistant Professor, Department of Nephrology, Katuri Medical College, Guntur, Andhra Pradesh, India
Corresponding Author: Dr. Varalakshmidevi B Assistant Professor, Department of Nephrology, Katuri Medical College, Guntur, Andhra Pradesh, India

\section{Ig M nephropathy: A clinicopathological study}

\author{
Dr. Varalakshmidevi B
}

DOI: https://doi.org/10.22271/27069567.2021.v3.i1a.97

\begin{abstract}
Introduction: Most studies of IgM nephropathy have consisted of patients with nephrotic syndrome (NS). However, IgM nephropathy is also associated with hematuria and asymptomatic proteinuria. In the long-term follow-up approximately one-third of the NS patients found to develop renal failure of some degree, half the patients experienced hypertension and some developed focal segmental glomerular sclerosis (FSGS) ${ }^{[1-2]}$. There are only few studies have been reported on the populationbased incidence and prevalence, mode of presentation, immunopathological features, pattern of steroid response, and the long-term prognosis of IgM nephropathy in either children or adults.

Objective: To describe the clinical and immunopathological features of $\operatorname{IgM}$ nephropathy and its response to steroids and its progression to renal failure.

Method: This is a descriptive, retrospective study carried out in SVIMS and Katuri hospitals, where the clinical records of patients with IgMN were analysed.

Results: From a total number of 2000 renal biopsies, performed during this period, we encountered 45 patients $(2.25 \%)$ of IgM nephropathy with renal biopsy proof. The number of adults were 35 (77.7\%), the number of males were $24(53.3 \%)$. The mean age was 28 years and age range was one year to 64 years; male to female ratio was 1.2:1. The renal syndrome presentation at the first instance was NS in all 45. In addition to NS the other clinical manifestations included hypertension in six, hematuria in eight, and elevated serum creatinine in four patients.

Conclusion: In conclusion, IgM nephropathy is an important cause of NS in both children and adults. It shows a spectrum of morphologic changes ranging from minor changes to FSGS. The diagnosis depends on IF. Although a poor clinical response to steroid therapy distinguished this disease from minimal change disease, in our patients the steroid sensitivity was greater.
\end{abstract}

Keywords: Ig M nephropathy, clinicopathological, SVIMS

\section{Introduction}

Most studies of IgM nephropathy have consisted of patients with nephrotic syndrome (NS). However, IgM nephropathy is also associated with hematuria and asymptomatic proteinuria. It appears that there are two sub- groups of $\operatorname{IgM}$ nephropathy in presentation: a sub-group with predominance of men usually manifest NS associated with progressive disease and the other group mainly seen in women with microscopic hematuria with favorable prognosis. In the long-term follow-up approximately one-third of the NS patients found to develop renal failure of some degree, half the patients experienced hypertension and some developed focal segmental glomerular sclerosis (FSGS) ${ }^{[1-2]}$

\section{Material and Methods \\ Study Design}

A retrospective descriptive study was done from the records of authors' institution.

\section{Study Population}

The patients information obtained from the review records between 1994 and 2019. From a total number of 2000 renal biopsies performed during this period, we identified 45 patients of IgM nephropathy.

\section{Analysis of the parameters}

Clinical history and findings of age, sex, hypertension, quantitative proteinuria, and serum creatinine ( $\mathrm{SCr}$ ) were considered for evaluation. The diagnosis of IgM nephropathy was determined by the presence of typical light microscopy features and glomerular $\operatorname{IgM}$ deposition on immunofluorescence (IF). Electron microscopy is not available at authors' institution. 
Follow ups in case records also evaluated to know the response to treatment and progression of disease.

\section{Study Procedure}

The patients information obtained from the review records between 1994 and 2019.

From a total number of 2000 renal biopsies performed during this period, we identified 45 patients of $\operatorname{IgM}$ nephropathy.

Clinical history and findings of age, sex, hypertension, quantitative proteinuria, and serum creatinine $(\mathrm{SCr})$ were considered for evaluation. The diagnosis of $\operatorname{IgM}$ nephropathy was determined by the presence of typical light microscopy features and glomerular IgM deposition on immunofluorescence (IF). All patients managed as follows, the first episode was treated with prednisolone of 0.5 to 2.0 $\mathrm{mg} / \mathrm{kg} /$ day for six months. If necessary, the prednisolone dose was reduced to $0.5 \mathrm{mg} / \mathrm{kg} /$ day, but only after three months. The treatment of steroid-dependent $\operatorname{IgM}$ nephropathy was with cyclophosphamide, $2 \mathrm{mg} / \mathrm{kg} / \mathrm{d}$ for 12 weeks. The treatment of steroid-resistant IgM nephropathy was with cyclosporine at $6 \mathrm{mg} / \mathrm{kg} /$ day for children or 5 $\mathrm{mg} / \mathrm{kg} /$ day for adults for at least six months. All patients were treated with levamisole $2.5 \mathrm{mg} / \mathrm{kg}$ /alternate day for 18 months after remission. Follow ups in case records also evaluated to know the response to treatment and progression of the disease.

\section{Results}

From a total number of 2000 renal biopsies, performed during this period, we encountered 45 patients $(2.25 \%)$ of IgM nephropathy with renal biopsy proof. The number of adults were $35(77.7 \%)$, the number of males were 24 $(53.3 \%)$. The mean age was 28 years and age range was one year to 64 years; male to female ratio was $1.2: 1$. The renal syndrome presentation at the first instance was NS in all 45 . In addition to NS the other clinical manifestations included hypertension in six, hematuria in eight, and elevated serum creatinine in four patients.

The pattern of response to steroids in our patients included, steroid-sensitive NS (SSNS) in 33, steroid resistant NS in five, steroid- dependent NS in seven. Among 33 patients of SSNS, 19 were infrequent relapsers, seven were frequent relapsers and another seven never had a relapse.

The details of renal biopsy findings were tabulated (Table 1). At the end of follow-up for a mean duration of $3.4 \pm 0.6$ years, 40 patients achieved remission and five patients had reached chronic kidney disease with serum creatinine 3.8 , 4.0, 4.5 and $5.1 \mathrm{mg} / \mathrm{dL}$.

Table 1: Renal biopsy findings in 45 patients of IgM nephropathy

\begin{tabular}{|c|c|}
\hline Biopsy findings & Number of patients (\%) $(\boldsymbol{n}=\mathbf{4 5})$ \\
\hline Light microscopy & $6(13.3)$ \\
\hline Normal glomeruli & $6(13.3)$ \\
\hline Mild focal and segmental mesangial hypercellularity & $15(33.3)$ \\
\hline Diffuse mesangial hypercellularity & $13(28.8)$ \\
\hline Mesangial matrix expansion & $22(48.8)$ \\
\hline Interstitial inflammation & 0 \\
\hline Interstitial fibrosis & 0 \\
\hline Tubular atrophy & $4(8.8)$ \\
\hline Hyaline arteriosclerosis & $35(77.7)$ \\
\hline Immunofluorescence microscopy & $6(13.3)$ \\
\hline IgM & $4(8.8)$ \\
\hline IgM + C3 & 0 \\
\hline IgM + IgG & IgA \\
\hline
\end{tabular}

\section{Discussion}

There are only few studies have been reported on the population-based incidence and pre- valence, mode of presentation, immunopathologic features, pattern of steroid response, and the long-term prognosis of IgM nephropathy in either children or adults. There is a wide variation in the prevalence of IgM nephropathy and its long-term prognosis. The reason for this is unclear. Two studies found that frequencies of $2 \%$ and $6.1 \%$, respectively in their biopsy series ${ }^{[3-4]}$.

In our study, the adults were more in number than children and a similar observation was reported in the earlier studies [2-7]. We did not find sex predilection. In earlier studies, predomi- nance of either sex was reported.

In the earlier reports, steroid resistance was reported in $28 \%$ ${ }^{[5]}$ and steroid dependence in about $33 \%{ }^{[1]}$. While, in our study, among the NS patients, steroid-sensitive were $74 \%$, ste- roid resistance in $10 \%$ and steroid dependence in $16 \%$. In our study, $15 \%$ had raised $\mathrm{SCr}$ at presentation while it was reported as $23 \%$ and $27 \%$ in earlier studies ${ }^{[1]}$.
In renal histopathology, the majority had matrix expansion, interstitial inflammation with mono-nuclear infiltration with IgM deposits on IF. FSGS was found in only one patient. Similar observation was reported in earlier studies ${ }^{[1,8-10]}$.

The clinical course and prognosis of IgMN nephropathy were very variable. In part the variation in these studies was due to variable follow-up duration In the largest and longest follow-up study, renal failure was observed in 35\% of cases at 15 years after initial biopsy, and $23 \%$ of patients went on to develop end- stage renal disease ${ }^{[2,11-16]}$.

\section{Conclusion}

In conclusion, IgM nephropathy is an important cause of NS in both children and adults. It shows a spectrum of morphologic changes ranging from minor changes to FSGS. The diagnosis depends on IF. Although a poor clinical response to steroid therapy distinguished this disease from minimal change disease, in our patients the steroid sensitivity was greater. 


\section{Acknowledgement}

The author is thankful to Department of Nephrology, Katuri Medical College for providing all the facilities to carry out this work.

Conflict of interest: None declared. Financial support: Nil

\section{References}

1. Mokhtar GA. IgM nephropathy: Clinical picture and pathological findings in 36 patients. Saudi J Kidney Dis Transpl. 2011;22:969-75.

2. Myllymäki J, Saha H, Mustonen J, Helin H, Pasternack A. IgM nephropathy: Clinical picture and long-term prognosis. Am J Kidney Dis. 2003;41:343-50.

3. Cohen AH, Border WA, Glassock RJ. Nehprotic syndrome with glomerular mesangial IgM deposits. Lab Invest. 1978;38:610-9.

4. Bhasin HK, Abuelo JG, Nayak R, Esparza AR. Mesangial proliferative glomerulonephritis. Lab Invest. 1978;39:21-9.

5. Border WA. Distinguishing minimal-change disease from mesangial disorders. Kidney Int. 1988;34:419-34.

6. Hsu HC, Chen WY, Lin GJ, et al. Clinical and immunopathologic study of mesangial IgM nephropathy: report of 41 cases. Histopathology. 1984;8:435-46.

7. Pardo V, Riesgo I, Zilleruelo G, Strauss J. The clinical significance of mesangial IgM deposits and mesangial hypercellularity in minimal change nephrotic syndrome. Am J Kidney Dis. 1984;3:264-9.

8. Ji-Yun Y, Melvin T, Sibley R, Michael AF. No evidence for a specific role of IgM in mesangial proliferation of idiopathic nephrotic syndrome. Kidney Int. 1984;25:100-6.

9. Kopolovic J, Shvil Y, Pomeranz A, Ron N, Rubinger D, Oren R. IgM nephropathy: morphological study related to clinical findings. Am J Nephrol. 1987;7:275-80.

10. Border WA. Distinguishing minimal change disease from mesangial disorders. Kidney Int. 1988;34:419-24.

11. Habib R, Girardin E, Gagnadoux M-F, Hinglais N, Levy M, Broyer M. Immunopathological findings in idiopathic nephrosis: clinical significance of glomerular “immune deposits". Pediatr Nephrol. 1988;2:402-8.

12. Mokhtar GA. IgM nephropathy: clinical features and pathological findings in 36 patients. Saudi J Kidney Dis Transpl. 2011;22:969-75.

13. Al Eisa A, Carner JE, Lirenman DS, Magil AB. Childhood IgM nephropathy: comparison with minimal change disease. Nephron. 1996;72:37-43.

14. Saha H, Mustonen J, Pasternack A, Helin H. Clinical follow up of 54 patients with IgM nephropathy. Am J Nephrol. 1989;9:124-8.

15. Kishimoto H, Arakawa M. Clinico-pathological characterization of mesangial proliferative glomerulonephritis with predominant deposition of IgM. Clin Exp Nephrol. 1999;3:110-5.

16. Donia AF, Sobh MA, Moustafa FE, Bakr MA, Foda MA. Clinical significance and long term evolution of minimal change histopathologic variants and of $\operatorname{IgM}$ nephropathy among Egyptians. J Nephrol. 2000;13:275-81. 